\title{
O impacto dos media no medo de contrair COVID-19
}

\section{The impact of the media on the fear of contracting COVID-19}

\author{
Sofia Figueiredo 1 \\ Ilda Massano-Cardoso $(1,2,3)$ \\ (1) Instituto Superior Miguel Torga, Coimbra, Portugal \\ (2) Universidade de Coimbra, Faculdade de Medicina, Portugal \\ (3) Universidade de Coimbra, Faculdade de Economia, Centro de Estudos e Investigação em Saúde da Universidade de Coimbra (CEISUC), Portugal
}

Recebido: 22/10/2021; Revisto: 09/11/2021; Aceite: 18/11/2021.

\begin{abstract}
Resumo
Objetivo: Pretendeu avaliar-se o impacto dos media no medo de contrair COVID-19. A confiança na informação associada a cada meio de comunicação, apreensão relativamente à divulgação diária das estatísticas, apreensão sentida com as medidas de prevenção e advertências veiculadas pelos meios de comunicação social, frequência de consumo de notícias, avaliação dos media na clarificação e informação sobre a pandemia e o medo de contrair a doença foram o foco deste estudo. Métodos: Amostra constituída por 349 indivíduos que completaram um Questionário Sociodemográfico, um Questionário de Opinião sobre os Media e o Fear of Contracting Covid Scale 19 (FCCS) através de uma plataforma online. Resultados: O medo de contrair COVID-19 foi superior nas mulheres em relação aos homens. Os participantes com mais medo de contrair a doença foram os que mais confiaram na informação da televisão, jornais e rádio, mas não das redes sociais. Scores mais elevados na FCCS corresponderam a valores mais elevados na clarificação e informação transmitida pelos media. A apreensão sentida com a divulgação diária de novos casos e novas medidas de prevenção/advertências pelos media, frequência de consumo de notícias, e avaliação dos media na clarificação e informação foram preditores do medo de contrair COVID-19. Conclusões: Os media demonstraram ser fundamentais na consciencialização e sensibilização da população.
\end{abstract}

Palavras-Chave: COVID-19; Impacto; Media; Medo.

\section{DI\&D | ISMT}

rpics@ismt.pt

https://rpics.ismt.pt distribuído sob a Licença Creative Commons Attribution, que permite uso, distribuição e reprodução sem restrições em qualquer meio, desde que o trabalho original seja devidamente citado.
Sofia Figueiredo

R. Augusta, 49, 3000-631, Coimbra.

Portugal

Telef.: 969831546

E-mail:sofia@ismt.pt 


\begin{abstract}
Objective: The current study sought to assess the mass media impact on the fear of contracting COVID-19. We focused on people's trust in information associated with media type, worry regarding daily reports of statistics, concerns about prevention measures and warnings in the media, news consumption frequency, evaluation of the media in explaining and informing about the pandemic, and the fear of contracting the disease. Methods: The sample comprised 349 participants who completed online a Sociodemographic Questionnaire, a Mass Media Opinion Questionnaire, and the Fear of Contracting Covid-19 Scale (FCCS). Results: Fear of contracting COVID-19 was higher in women compared to men. Participants with higher fear of contracting the disease trusted more in the information provided by television, newspapers, and radio, but not social media. Higher scores on the FCCS were associated with higher scores on clarification, awareness, and information conveyed by media. Feelings regarding daily reports of COVID-19 and concerns about prevention measures and warnings in the media, news frequency consumption, and media evaluation in explaining and informing about the pandemic were predictors of fear of contracting COVID-19. Conclusions: Mass media proved to be fundamental in raising awareness and sensitization of the population.
\end{abstract}

Keywords: COVID-19; Fear; Impact; Media.

\title{
Introdução
}

\section{O Impacto dos Media no Atual Contexto Pandémico}

O atual contexto pandémico ficará na história universal, não só pelas questões da saúde pública, mas porque convocou a população a refletir e redefinir conceitos e paradigmas de uma sociedade globalizada e mediatizada.

O mundo parou em março de 2020, confinou a maioria dos setores e imperaram as tecnologias e aplicações que juntaram famílias, promoveram o teletrabalho e o ensino à distância (Dey et al., 2020). Mais do que nunca, a informação, a necessidade de perceber a nova doença que acabara de eclodir, o medo de a contrair e a ansiedade sobre as consequências, a nível laboral, social, político e económico, colocou a sociedade atenta às notícias (Ytre-Arne, 2021). O consumo das notícias intensificou-se e as pessoas tornaram-se mais dependentes da informação permanente e da triagem das coisas, dos factos. Em Portugal, segundo a Marktest (2020), na semana de 2 a 8 de março de 2020, registou-se um forte crescimento no acesso a páginas de conteúdos informativos, assumindo as páginas das marcas dos canais televisivos e radiofónicos, a segunda posição. O mesmo artigo indica que no final da segunda semana desse mesmo mês, o acesso a estas mesmas páginas de informação subiu 78\%.

Os meios de comunicação social influenciam e conseguem incutir na população uma forma de agir e de pensar. Luhmann (2021, p. 15) afirma que "aquilo que sabemos sobre a nossa sociedade, ou mesmo sobre o mundo no qual vivemos, o sabemos pelos meios de comunicação." São meios de comunicação social que dizem sobre o que pensar, como pensar e quais os temas do dia, assim o público tenha confiança no que ouve, lê ou assiste nas notícias, o que decorre da análise ao estudo de Edelman (2020). De acordo com um estudo da Edelman (2020), constata-se que a necessidade de confiança nos meios de comunicação social, durante a COVID-19, é evidenciada pelo aumento de pesquisa de informação fidedigna sobre o tema.

Seguindo as conclusões de um estudo realizado em 2020 pela Marktest e divulgado no jornal online Observador (Barbosa, 2020), o grau de confiança perante os noticiários de televisão (TV), neste período, obteve um valor de $86 \%$. Nesta pesquisa, todo o ranking deu confiança aos outros media mainstream, mas 
no campo oposto da fiabilidade constam as redes sociais. Este estudo evidenciou a preocupação dos portugueses em vários domínios. Na saúde, por exemplo, o estudo de Barbosa (2020) revela que "80\% receia ficar ou ver um familiar infetado e $72 \%$ tem medo de ficar doente". Preocupações estas que se vão interiorizando e adquirindo à medida que o tempo de exposição às notícias vai decorrendo. Num contexto pandémico, onde a incerteza se instala, a proliferação de informação nos media pode gerar um clima de constante competição, provocando no cidadão uma enorme dificuldade em distinguir o essencial do assessório e o verdadeiro do falso, promovendo assim o medo (Cabrera et al., 2020).

O processo de aquisição e transmissão de informação resume-se à aceitação de forma simples e objetiva do esquema de influência dos media. Os construtores da mensagem querem ensinar o que os recetores querem aprender com as notícias. A TV tem a capacidade de explicar todo o processo que é o que mais prende o espetador (Potter, 2012). Resultados de um estudo realizado por Hua e Shaw (2020) traduzem o uso dos media durante a pandemia, nomeadamente a televisão, atribuindo-lhe um forte papel na aquisição de informação depois da disseminação do Coronavírus, uma vez que a televisão permite a informação em tempo real.

O papel pedagógico dos media, numa questão como a cobertura intensiva dos casos de doença COVID19, é inegável que ficou bem vincado neste período e serviu também para responsabilizar e sensibilizar o público para a adoção de medidas de segurança e de proteção individual (Saud et al., 2020). No cenário pandémico, a atenção às notícias foi uma questão de esclarecimento e sobrevivência, conclusão que decorre do estudo de (Bernardino et al., 2016) que sugere ser nesta interação que existe espaço para se criarem e gerarem emoções, para além de influenciar estados emocionais e sentimentos de bem-estar. Através dos media, o indivíduo toma decisões, das mais simples às mais complexas. É inegável que a exagerada exposição às notícias pode conduzir à sobrecarga e ansiedade, aumentado o medo. A dificuldade em avaliar e selecionar informação relevante aumenta à medida que estão disponíveis mais fontes e conteúdos diversos. A sobrecarga de informação, Information Overload, é uma consequência (Schmitt et al., 2017).

Refletir sobre o impacto dos media implica considerar a sua relevância e assumir a maior evidência do jornalismo: informar e esclarecer a sociedade sobre a atualidade. Comummente percecionado como um elemento vital nas sociedades e nas democracias, o jornalismo tem sido, por muitos, considerado como o quarto poder. Aceitando esta relevância dos media e seguindo Kovach e Rosenstiel (2005), a finalidade do jornalismo, independentemente da técnica e suas especificidades normativas, é definida pelo papel que as notícias representam na vida das pessoas. A confiança na informação e a credibilidade são imprescindíveis para o impacto positivo dos media Freundt (2009). O mesmo autor afirma que qualquer erro que possa por em causa a perceção da qualidade dos conteúdos e da transparência dos media põe em risco a confiança do público.

\section{Medo e Informação}

Seguindo o estudo de Ahmad et al. (2021), qualquer crise, como uma pandemia, têm tanto oportunidades como ameaças. E, revelam os autores no estudo, sem uma intervenção adequada, nestas situações, é fácil desenvolver ansiedade e medo. O medo é um sentimento normal e adaptativo. Rosen e Schulkin (1998, p. 325) definem-no como "o estado de medo adaptativo e de ansiedade" e ainda que o medo seja uma 
resposta a um "evento potencialmente perigoso". O medo é um mecanismo de defesa animal adaptativo e fundamental para a sobrevivência (Wang et al., 2019).

Em contexto de pandemia, nomeadamente em contexto de transmissão, o medo de ser infetado e as suas consequências podem assumir dimensões exuberantes e tendem a afetar o bem-estar psicológico das pessoas (Salvaterra \& Chora, 2021). Essa exuberância pode dar origem ao pânico social que se carateriza por um sentimento redimensionado a uma escala global que, de acordo com Hossain et al. (2020) desencadeia sentimentos e comportamentos como angústia, insegurança e medo. Se por um lado, as autoridades de saúde e os veículos governamentais possuem um papel importante e inesgotável sobre as práticas e medidas a adotar no que concerne à saúde das populações, por outro, é através da ferramenta da comunicação que essas práticas são difundidas (Hung et al., 2021). De acordo com Wilder-Smith e Freedman (2020) são os media que muitas vezes tranquilizam a população e elucidam sobre os motivos do isolamento social, conseguindo assegurar um efeito positivo e até sanando e prevenindo o pânico. A atitude de isolamento social está muitas vezes associada ao altruísmo, permitindo assim, através da informação, assumir comportamentos de adesão e reduzir consideravelmente o seu impacto psicológico (Rubin \& Wessely, 2020).

Na perspetiva de Wang et al. (2020), torna-se essencial informar a população sobre os factos verídicos, transmitindo declarações oficiais com a finalidade de reduzir as consequências negativas. Reduzir o medo e promover estratégias de promoção de saúde mental dos indivíduos pode ser um contributo fundamental por parte dos meios de comunicação social. A redução da ansiedade pode passar pelo esclarecimento de dúvidas sobre a pandemia, a apresentação das estatísticas, a divulgação de resultados positivos, que ainda assim existem, assentes na transparência da comunicação. Com efeito, a facilidade de acesso às novas tecnologias de comunicação, bem como a transmissão de informações sensacionalistas, imprecisas ou falsas (fake news) estão associadas ao aumento das reações sociais prejudiciais, como raiva e comportamento agressivo (Wang et al., 2019).

A "infodemia", assim chamada a atual pandemia pela torrente de notícias e o uso de expressões como: "os serviços estão a entrar em colapso", "os números estão a aumentar", "vem aí a terceira vaga" terão tido reflexo no medo e na apreensão por parte da população, pela exposição constante às mesmas. Tedros Adhanom Ghebreyesus, Diretor Geral da OMS (2020), referiu na conferência de Munich, a 15 de fevereiro que "We're not just fighting an epidemic; we're fighting an infodemic". As afirmações denotam a necessidade de desafio no controlo da informação disseminada, pela sua tão grande vulnerabilidade e que, tal como o vírus, viaja com as pessoas e circula de forma rápida. No entanto, a procura de informação é uma parte inerente do quotidiano dos indivíduos. À medida que procuram informação, fazem-no para alargar a sua compreensão sobre um determinado acontecimento e para satisfazer as suas necessidades (Schmitt et al., 2017).

\section{Estudo Presente}

Nesse sentido, o impacto dos media no medo de contrair COVID-19 merece ser analisado em diferentes vertentes. O principal objetivo do presente trabalho foi estudar e avaliar a confiança na generalidade da informação transmitida pelos media, especificamente associada a cada meio de comunicação, perceção sobre a divulgação diária das estatísticas associadas à COVID-19, frequência com que recorre às notícias, classificação do contributo dos media na clarificação e esclarecimento e informação sobre a pandemia e 
por fim avaliar o medo de contrair a doença. Adicionalmente, foi explorada a relação do medo com as variáveis sociodemográficas idade e sexo.

\section{Método}

\section{Participantes}

A presente amostra foi composta por 349 indivíduos sendo 77,4\% ( $n=270)$ do sexo feminino e 22,6\% ( $n$ = 79) do sexo masculino. A média de idades dos participantes foi de 34,43 anos ( $D P=12,83$ ). $O$ estado civil dominante no estudo foi casado(a)/união de facto com 173 indivíduos (49,6\%), seguido de solteiro com 156 (44,7\%) e o menos representado o divorciado ( $n=19 ; 5,4 \%)$. No que respeita à distribuição geográfica de residência dos participantes 183 (52,4\%) residiam em meio urbano e 166 (47,6\%) em meio rural. A ocupação dominante entre os inquiridos foi trabalhador(a), que representou $65,3 \%(n=228)$, seguido de estudante ( $n=91 ; 26,1 \%)$, desempregado $(n=15 ; 4,3 \%)$, estudante/trabalhador $(n=9 ; 2,6 \%)$ e reformado ( $n=6 ; 1,7 \%$ ). A maioria dos inquiridos tinha nacionalidade portuguesa, representando 342 respostas $(98,0 \%)$.

\section{Instrumentos}

\section{Questionário Sociodemográfico}

O questionário sociodemográfico englobou as seguintes variáveis: idade, género, nacionalidade, ocupação, zona de residência e estado civil.

\section{Questionário Sobre o Impacto dos Media no Medo de Contrair COVID-19 (QIM-MCC-19)}

O QIM-MCC-19 é um instrumento de autorresposta que foi criado para este estudo com o objetivo de avaliar o impacto dos media no medo de contrair COVID-19. Este questionário ficou constituído por três partes. Uma parte incluiu uma questão sobre se o participante já tinha estado ou não infetado com SarsCov2 e outra questão sobre se já tinha realizado teste à COVID-19. Estas duas questões não foram exploradas no presente estudo. A segunda parte incluiu seis questões de resposta direta e de opinião sobre a confiança sentida em relação à generalidade da informação, nomeadamente da televisão, rádio, jornais e redes sociais através de uma escala de Likert com cinco opções de resposta ["Nada confiável" (1) a "Plenamente confiável" (5)]. A terceira parte incluiu quatro questões:

1) Apreensão dos participantes relativamente à divulgação diária das estatísticas associadas à COVID19 (3 categorias de resposta: "Nada apreensivo", "Apreensivo" e "Indiferente");

2) Apreensão sentida com as medidas de prevenção e advertências veiculadas pelos meios de comunicação social (3 categorias de resposta: "Nada apreensivo", "Apreensivo" e "Indiferente");

3) Frequência com que recorria às notícias (4 categorias de resposta: "Nunca vejo notícias", "Normal", "Vejo mais do que deveria", "Considero extremamente exagerado");

4) Apreciação sobre se os media contribuem para a clarificação e informação sobre a pandemia (5 categorias de resposta: "Nada eficaz" a "Muito eficaz"). 


\section{Fear of contracting Covid 19 Scale (FCCS)}

A FCCS (Trindade \& Ferreira, 2020) é uma escala desenvolvida especificamente para o estudo no âmbito da COVID-19. Foi baseada no Fear of Aids Scale (Arrindell et al., 1989) e pede aos participantes que indiquem o nível de medo/preocupação que dispõem em relação a cada situação apresentada. Os nove itens desta escala são avaliados numa escala de cinco pontos de 1 (nenhum medo) a 5 (medo extremo), com as pontuações mais altas significando mais medo e preocupação em contrair COVID-19. A pontuação total varia entre 9 e 45. O alfa de Cronbach da escala utilizada revelou-se excelente com um valor de 0,91. No presente estudo o cálculo da consistência interna da escala foi de $\alpha=0,90$. Foi ainda confirmada a estrutura fatorial da escala com recurso à Análise das Componentes Principais que apresentou valores excelentes no cálculo do teste Kaiser-Meyer-Olkin $(0,87)$ e o teste de esfericidade de Bartlett $\left(\chi^{2}(36)=\right.$ 1783,48; $p<0,001$ ) (Hutcheson \& Sofroniou, 1999).

\section{Procedimentos}

Foi solicitada via correio eletrónico a autorização do autor para a utilização do instrumento de autorresposta referido anteriormente. Posteriormente, foi criada na plataforma Google Forms o questionário para a presente investigação. O recrutamento foi efetuado através das redes sociais e e-mail, usando processo de amostragem por snowball entre a rede de contactos dos investigadores. A recolha de dados foi efetuada online entre outubro e dezembro de 2020, sendo disponibilizado um link com acesso ao questionário (https://bit.ly/3xrrhAS). Os participantes, sempre que acedessem ao link, eram informados do âmbito e objetivo do estudo, tendo sido assegurado o anonimato e confidencialidade dos dados através de um consentimento informado. Adicionalmente, foi criado um endereço de correio eletrónico para esclarecimento de dúvidas que pudessem surgir. Os critérios de inclusão definidos consistiram em ter idade igual ou superior a 18 anos.

\section{Análise Estatística}

Os dados foram recolhidos através da plataforma Google Forms, tendo sido transferidos para um ficheiro Excel e posteriormente exportados para uma base de dados no software estatístico IBM SPSS Statistics v.26. Recorremos à estatística descritiva, nomeadamente, moda, média e respetivos desvios padrão das variáveis em estudo.

Para calcular a eventual associação entre variáveis de natureza qualitativa nominal recorremos ao cálculo do Qui-quadrado $\left(\chi^{2}\right)$, à correlação de Pearson para a associação entre variáveis de natureza quantitativa, ao cálculo do teste $t$ de Student e ANOVA para verificar diferenças entre grupos, ao teste $t$ para uma amostra para comparar os valores obtidos neste estudo com os valores teóricos e a uma análise de regressão linear múltipla hierárquica para examinar a contribuição dos diferentes preditores na explicação do medo de contrair COVID-19 (FCCS).

O tamanho do efeito para a análise das diferenças entre dois grupos $-d$ de Cohen - foi calculado relativamente ao total da FCCS (Cohen et al., 2003). De acordo com o recomendado pelo autor, valores entre 0,20 e 0,49 são considerados pequenos, entre 0,50 e 0,79 médios e os superiores a 0,80 são grandes. 
O tamanho do efeito na comparação de mais de dois grupos foi analisado através do eta-parcial ao quadrado $\left(\eta_{p}^{2}\right)$, sendo interpretados como valores de 0,01 pequenos, 0,06 médios e 0,14 grandes (Tabachnick \& Fidell, 2007). Quanto à análise correlacional, valores de 0,50 a 1,00 são considerados grandes; de 0,30 a 0,49 moderados e de 0,10 a 0,29 pequenos (Cohen, 1988). Na regressão linear múltipla hierárquica, recorreu-se ao $R$-quadrado ajustado que quanto mais alto, maior a força da predição (Hays, 1963).

Recorremos ainda ao cálculo da consistência interna através da análise do alfa de Cronbach.

\section{Resultados}

A média do score total da FCCS foi de 30,64 ( $D P=6,97$ ). Ao comparar os valores médios obtidos na presente investigação com os reportados por Trindade e Ferreira (2020) numa amostra de pacientes com doença inflamatória do intestino, verificou-se que a média do medo de contrair COVID-19 obtida no nosso estudo foi inferior de forma estatisticamente significativa ( $t=-13,26 ; p<0,001)$.

A análise da eventual relação entre a variável idade e o medo de contrair COVID-19 avaliado através da FCCS revelou uma correlação estatisticamente significativa pequena $(r=-0,11 ; p=0,043)$, sendo que foram os indivíduos mais novos os que apresentaram um resultado mais elevado de medo de contrair a doença.

Explorado o efeito do género no medo de contrair COVID-19 avaliado através da FCCS, verificaram-se existir diferenças estatisticamente significativas. Assim, o género feminino obteve um resultado superior $(M=31,52 ; D P=6,64)$ ao género masculino $(M=27,6 ; D P=7,28)\left(t_{(347)}=4,45 ; p<0,001\right)$. O tamanho do efeito foi médio $(d=0,57)$.

O medo de contrair COVID-19 (FCCS) revelou estar associado de forma significativa com a confiança nos meios de comunicação social avaliada através do QIM-MCC-19, nas opções televisão $(r=0,17 ; p=0,002)$, rádio $(r=0,13 ; p=0,01)$ e jornais $(r=0,19 ; p<0,001)$, não tendo sido significativa nas redes sociais $(r=$ $0,10 ; p=0,63)$. O tamanho do efeito foi pequeno $\left(r^{2}=2,9 \% ; r^{2}=1,7 \%, r^{2}=3,6 \%\right.$, respetivamente).

A média do score total da FCCS mostrou diferenças estatisticamente significativas quando comparada consoante o grau apreensão causada pela divulgação do número de novos casos diários (QIM-MCC-19), traduzida em "nada apreensivo", "apreensivo" ou "indiferente" $\left(F_{(2,346)}=39,83 ; p<0,001 ; \eta_{p}^{2}=0,19\right)$. Os indivíduos que consideraram ser "indiferentes" à divulgação do número de novos casos apresentaram um score médio superior aos demais $(M=32,84 ; D P=5,94)$. Seguiram-se os indivíduos que responderam "apreensivo" ( $M=27,72 ; D P=7,02)$ e por último os que consideraram ser "nada apreensivos" $(M=6,87$; $D P=6,87)$.

O medo de contrair COVID-19 revelou diferenças estatisticamente significativas $\left(F_{(2,346)}=28,26 ; p<\right.$ 0,$\left.001 ; \eta_{p}^{2}=0,14\right)$ consoante o que os participantes sentiam em relação às novas medidas de prevenção e advertências veiculadas pelos meios de comunicação social. Assim, os indivíduos que apresentaram mais medo foram os que consideraram ser "indiferentes" $(M=33,30 ; D P=5,66)$, seguidos dos que responderam "nada apreensivo" ( $M=28,09 ; D P=7,16)$ e "apreensivo" $(M=28,08 ; D P=7,42)$. 
O medo de contrair COVID-16 variou consoante a autoperceção da frequência de consumo das notícias. Os indivíduos que consideraram que "Vejo mais do que deveria" ( $n=75)$ apresentaram scores totais mais elevados na FCSS $(M=33,70 ; D P=5,77)$ comparativamente com os que consideraram ter uma frequência "Normal" ( $n=227 ; M=30,38 ; D P=6,64)$. Com valores aproximados estiveram os inquiridos que consideraram "Nunca vejo notícias" ( $n=21 ; M=27,06$; $D P=7,62$ ) e os que "Considero extremamente exagerado" ( $n=26 ; M=27,04 ; D P=8,92$ ). Assim os indivíduos que apresentaram mais medo foram os que consideraram ter maior frequência do consumo de notícias do que o normal e os que consideram fazer um consumo de notícias "normal" $\left(F_{(2,346)}=9,77 ; p<0,001 ; \eta_{p}^{2}=0,08\right)$.

Os inquiridos que apresentaram scores mais elevados na escala do FCCS foram os que consideraram que, de uma maneira geral, os media contribuíam na clarificação e informação sobre a atual pandemia $(r=0,24$; $p<0,001)$.

Com o intuito de explorar quais os preditores do medo de contrair COVID-19, procedeu-se ao cálculo da regressão linear múltipla hierárquica após confirmação do cumprimento dos seus pressupostos (tamanho da amostra adequado, ausência de multicolinearidade). A idade e o sexo (enquanto dummy) foram introduzidos no primeiro bloco; a confiança na TV, rádio e jornais, a apreensão sentida com a divulgação de novos casos, a apreensão com novas medidas de prevenção e advertências veiculadas pelos meios de comunicação social a frequência no consumo de notícias e a avaliação dos media quanto à clarificação e informação sobre a atual pandemia foram inseridas no segundo bloco. O primeiro bloco explicou 7,0\% da variância do medo de contrair COVID-19 e o segundo bloco contribuiu com 25,0\% para o aumento da variância, revelando-se ambos significativos (Tabela 1).

\section{Tabela 1}

Análise de Regressão Linear Múltipla Hierárquica dos Preditores do Medo de Contrair COVID-19

\begin{tabular}{|c|c|c|c|c|c|}
\hline Preditores & $r$ & $R_{\text {Ajustado }}^{2}$ & $F$ & $B$ & $t$ \\
\hline Modelo 1 & 0,27 & 0,07 & $13,04^{* * *}$ & & \\
\hline Idade & & & & $-0,12$ & $-2,21^{*}$ \\
\hline Sexo (dummy) & & & & $-0,24$ & $-4,63^{* * *}$ \\
\hline Modelo 2 & 0,52 & 0,25 & $13,46^{* * *}$ & & \\
\hline Idade & & & & $-0,11$ & $-2,29^{*}$ \\
\hline Sexo (dummy) & & & & $-0,17$ & $-3,56^{* * *}$ \\
\hline Confiança na TV & & & & 0,08 & 0,83 \\
\hline Confiança na Rádio & & & & $-0,15$ & $-1,46$ \\
\hline Confiança nos Jornais & & & & 0,14 & 1,68 \\
\hline Apreensão com divulgação de novos casos & & & & 0,24 & $4,36^{* * *}$ \\
\hline Apreensão com novas medidas/advertências veiculadas pelos media & & & & 0,19 & $3,37^{* * *}$ \\
\hline Frequência de consumo de notícias & & & & 0,05 & 0,96 \\
\hline Avaliação dos media quanto à clarificação e informação & & & & 0,14 & $2,45^{*}$ \\
\hline
\end{tabular}

Nota.

${ }^{*} p<0,05 .{ }^{* * *} p<0,001$. 
De referir que no primeiro modelo quer a idade, quer o sexo revelaram-se preditores do medo $(\beta=-0,12 ; p=$ $0,028)$. Já no segundo bloco, verificou-se que a idade $(\beta=-0,11 ; p=0,022)$, sexo $(\beta=-0,17 ; p<0,001)$, apreensão relativamente à divulgação de novos casos $(\beta=0,24 ; p<0,001)$, apreensão com novas medidas/advertências veiculadas pelos media $(\beta=0,19 ; p<0,001)$ e avaliação dos media quanto à clarificação e informação $(\beta=0,14 ; p=0,015)$ contribuíram para a predição do medo de contrair a COVID-19.

\section{Discussão}

Os resultados obtidos sugerem que o medo de contrair COVID-19, na população portuguesa revela preocupação e apreensão significativas ainda que no presente estudo os valores médios do score total da FCCS foram mais baixos quando comparado com o estudo original (Trindade \& Ferreira, 2020: $M=35.59$; $D P=6.21$, numa amostra de pacientes com doença inflamatória do intestino. Embora se assemelhem, verificou-se a existência de diferenças estatisticamente significativas no medo de contrair COVID-19. Atendendo que a cronicidade da doença é um fator de risco muito elevado para a COVID-19, sublinhamos a relevância do score obtido. Com efeito, sabe-se que durante as epidemias, o número de pessoas cuja saúde mental é afetada tende a ser superior ao número de pessoas afetadas pela infeção (Reardon, 2015). De acordo com a OMS, e como referido anteriormente, o neologismo "infodemia" remete para outro perigo das sociedades na era dos media sociais: a distorção da realidade ou factos frequentemente inventados que potenciam o medo (Cinelli et al., 2020).

A idade e o medo de contrair COVID-19, associaram-se de forma significativa sendo os indivíduos mais novos os que apresentaram um resultado mais elevado na FCCS.

O género feminino obteve um resultado superior no que respeita ao medo de contrair a doença comparativamente com o género masculino. De acordo com a literatura (Broche-Pérez et al., 2020), a probabilidade das mulheres com níveis de medo em comparação com os homens é de três vezes mais. $O$ mesmo estudo apresenta resultados que corroboram pesquisas internacionais que indicam uma maior vulnerabilidade psicológica nas mulheres durante a pandemia da COVID-19 (Broche-Pérez et al., 2020). No que respeita à relação do medo de contrair COVID-19 com a confiança, a associação significativa com a televisão, com os jornais e com a rádio são facilmente entendidos pela preferência dos portugueses. Estes resultados vão ao encontro dos publicados no relatório Digital News of Reuters Institute (Newman et al., 2020), da Universidade de Oxford, que revelaram que em 2019, Portugal foi o segundo país que mais confiava nas notícias e que a televisão era a fonte preferencial dos portugueses. Sabe-se que a televisualidade tem impacto e o seu conteúdo televisivo é credível. Afinal, quem não acredita e sente no que está a ver e a ouvir? Para lá de toda a edição associada aos processos de arranjo de imagem, esta é, aos olhos do público, um garante de credibilidade (Gradim, 2015, p. 73). Na verdade, a informação televisiva assume um conjunto de caraterísticas que a diferenciam e posicionam, superiormente, em relação aos outros meios: tanto a nível de alcance e audiência como a nível de capacidade de agendasetting (Wolf, 2006) e, pela sua ubiquidade que, em particular, torna as relações sociais vividas como espetáculo (Stratton, 2018). Pesquisas anteriores já tinham sugerido que a procura do meio está correlacionada com níveis de confiança. A investigação de Kalogeropoulos et al. (2019), baseada num 
inquérito aos utilizadores de notícias em trinta e cinco países, mostrou que a utilização de fontes noticiosas tradicionais está associada aos níveis elevados de confiança. Brewer e Pritchard (2008) pretenderam examinar a confiança na rádio. Os inquiridos expressaram níveis de confiança mais elevados na rádio informativa do que na rádio de entretenimento. Neste estudo, a confiança na rádio informativa era inferior à confiança na televisão, mas superior à confiança em sites; enquanto que a confiança na rádio de entretenimento era inferior à confiança na televisão ou nos jornais. Uma outra análise (Stamm et al., 1997) concluiu que, sob certas condições, as notícias de rádio podem produzir efeitos de magnitude comparável aos produzidos pelos noticiários e jornais de televisão. Os meios noticiosos tradicionais constituem a mais importante fonte de informação sobre a atualidade e a questão da confiança está, também, ligada à vontade dos cidadãos de aprenderem (Elvestad et al., 2018). Assim sendo, tal como Quintanilha et al. (2019), a confiança nas notícias deve ser considerada como uma extensão dos diferentes meios de comunicação da seletividade, imparcialidade, factualidade, credibilidade e objetividade das reportagens que divulgam. A confiança nos meios tradicionais de informação está intimamente associada à sua função social positiva, geradora de confiança. A necessidade de uma epistemologia do jornalismo, assente em conceitos como verdade, objetividade e factos torna-se essencial (Kovach \& Rosenstiel, 2005). O facto de as redes sociais não terem revelado expressão significativa pode estar associada ao descrédito destas plataformas como fonte de notícias e, também, como sugere Park et al. (2020), os níveis mais elevados de desconfiança geral nas notícias veiculadas nas redes, estão relacionados com o uso em demasia da partilha de notícias. De acordo com Shu et al. (2017), as redes sociais para o consumo de notícias são uma espada de dois gumes. Por um lado, o seu baixo custo, o seu fácil acesso e rápida disseminação de informação, levam as pessoas a procurar e a consumir notícias dos meios de comunicação social; por outro lado, permite a ampla difusão de notícias falsas. Desta forma, atendendo à situação que vivemos, cujo rigor da informação é fundamental, justifica-se que as redes sociais não possam ser um veículo de informação confiável. Neste contexto histórico, não devemos acreditar que a baixa confiança nos meios noticiosos é um estado de coisas inevitável, que deve ser aceite com relutância, como normal (Lewis, 2020). Esta mesma ideia é corroborada pelo estudo do Reuters Institute for the Study of Journalism (Newman et al., 2019), que se baseou num inquérito realizado online a 70 mil pessoas de 36 países. O estudo refere que em Portugal, mais de metade dos entrevistados (54\%) usam as redes sociais como fonte de notícias e apenas um quarto (24\%) pensa que as redes sociais podem contribuir para um trabalho na separação dos factos da ficção, em comparação com os $40 \%$ que acham que a comunicação social faz um bom trabalho. De acordo com o mesmo relatório os Portugueses confiam menos nas redes sociais como fonte de notícias, mas é o meio que mais usam.

O medo de contrair COVID-19 foi diferente consoante o grau de apreensão relativo à divulgação diária do número de casos, consoante a apreensão referente à divulgação de novas medidas de prevenção e advertência e conforme a frequência do consumo de notícias, o que nos remete para a necessidade de compreender estes fenómenos. Nesse sentido, as reações psicológicas a epidemias e pandemias sugerem que são múltiplos os fatores de vulnerabilidade psicológica que podem desempenhar um papel na resposta ao medo de contrair COVID-19, incluindo variáveis de diferenças individuais, tais como a intolerância da 
incerteza, a perceção da vulnerabilidade à doença, a preocupação e a ansiedade (Taylor, 2020). O papel dos media na clarificação e informação sobre a pandemia, tal como avaliado pelos inquiridos, relacionouse com o medo de contrair COVID-19. Assim, os inquiridos que apresentaram scores mais elevados na escala do FCCS foram os que consideraram que os media contribuíam para clarificar, esclarecer e informar sobre a atual pandemia. O mesmo se verificou num estudo de Aquino e Vieira (2020), utilizando o World Health Organization Well-Being Index (WHO-5), que pretendeu examinar a associação entre exposição a notícias e a perceção do papel dos media e bem-estar durante os estágios iniciais da pandemia de COVID19, no Brasil. Adicionalmente, o estudo refere que quanto mais se percebe a relevância dos meios de comunicação para informar a população sobre a gravidade da COVID-19, pior o grau de bem-estar medido com a WHO-5.

A apreensão sentida com a divulgação de novos casos, a apreensão com a divulgação de novas medidas/advertências, a frequência de consumo de notícias, e avaliação dos media quanto à clarificação e informação apresentaram-se como variáveis preditoras do medo de contrair COVID-19. O estudo de Dhir et al. (2018) demonstra a associação entre o acesso exagerado à exposição informativa e às notícias e a fadiga, podendo traduzir-se em medo, ansiedade e depressão. A confiança depositada nos meios de informação anteriormente elencados permite um maior conhecimento, maior perceção do risco e consequentemente, o aumento do medo. A confiança pode ser conseguida pelo estilo de jornalismo relacional que "coloca a construção e manutenção de relações com os públicos que serve normativamente no centro do seu trabalho" (Lewis, 2020, p. 347).

Não obstante das diversas limitações do estudo, consideramos que a principal limitação incidiu sobre o processo amostral. Ainda assim, o desequilíbrio da amostra por géneros foi semelhante ao estudo original (Trindade \& Ferreira, 2020). Assim, a técnica utilizada não-aleatória, em snowball, através da partilha online nas redes sociais, pode ter comprometido a representatividade da mesma.

\section{Conclusão}

O estudo contribuiu para uma compreensão global do papel dos media e do seu respetivo impacto num período onde o medo de contrair COVID-19 é uma realidade. A utilização do instrumento FCCS permitiu avaliar, com rigor, o medo e preocupação dos intervenientes neste estudo e associá-los ao impacto dos media. Desta forma, considerando que os media desenvolvem um papel pedagógico e de suporte na divulgação da informação à sociedade, os resultados encontrados constituem-se como um contributo fundamental para salvaguardar as necessidades de esclarecimento da população, elevando assim o importante papel da informação, em nome do interesse público. Conclui-se que a qualidade da informação jornalística está associada ao medo de contrair COVID-19, já que no modelo estudado, se verificou que variáveis relacionadas com os media (apreensão sentida com a divulgação de novos casos e de novas medidas de prevenção/advertências pelos media, a frequência de consumo de notícias, e avaliação dos media quanto à clarificação e informação) são preditores do medo de contrair a COVID-19. Assim, tornase imperioso insistir no rigor e precisão jornalística no que respeita à transmissão de informação em saúde. O presente estudo tem como principal contributo a possibilidade de sensibilizar os agentes da informação 
para a necessidade de disseminação de informação válida e fidedigna, sobretudo em contextos de pandemia.

Agradecimentos | Acknowledgements: Os autores agradecem a Francisca dos Reis, Maria Costa e Miguel Almeida, alunos do $1^{\circ}$ ciclo de estudos em jornalismo do ISMT.

Conflito de interesses | Conflict of interest: nenhum | none.

Fontes de financiamento | Funding sources: nenhuma | none.

Contributos: SF: Pesquisa bibliográfica; Revisão da literatura; escrita do manuscrito, revisão final. IMC: Desenho do estudo; Preparação das medidas e escrita do protocolo; Recrutamento dos participantes; Análise estatística; escrita e aprovação do manuscrito.

\section{Referências}

Ahmad, N. S., Hussain, Z., Abd Hamid, H. S., \& Khairani, A. Z. (2021). Roles of social media and counselling support in reducing anxiety among Malaysian during Covid-19 pandemic. International Journal of Disaster Risk Reduction, 63, Artigo 102456. https://doi.org/10.1016/j.ijdrr.2021.102456

Aquino, S. D., \& Vieira, L. S. (2020). Bem-estar e consumo de notícias durante a pandemia de COVID-19. Revista Fontes Documentais, 3, 165-174. https://bit.ly/3DzRsr7

Arrindell, W. A., Ross, M. W., Robert Bridges, K., van Hout, W., Hofman, A., \& Sanderman, R. (1989). Fear of aids: Are there replicable, invariant questionnaire dimensions? Advances in Behaviour Research and Therapy, 11(2), 69-115. https://doi.org/brm2pv

Barbosa, M. (2020, April 16). Covid-19. Estudo revela que portugueses confiam nos media e desconfiam das redes sociais. Observador. https://bit.ly/3x3MaSp

Bernardino, C., Ferreira, H., \& Chambel, T. (2016). Towards media for wellbeing. Proceedings of the ACM International Conference on Interactive Experiences for TV and Online Video, 171-177. https://doi.org/g6j3

Brewer, P. R., \& Pritchard, D. (2008). Ideology and public trust in radio as a source for local news. Journal of Radio \& Audio Media, 15(2), 124-135. https://doi.org/d8p5zq

Broche-Pérez, Y., Fernández-Fleites, Z., Jiménez-Puig, E., Fernández-Castillo, E., \& Rodríguez-Martin, B. C. (2020). Gender and fear of COVID-19 in a Cuban population sample. International Journal of Mental Health and Addiction. https://doi.org/gh7zd6

Cabrera, A., Martins, C., \& Cunha, I. (2020). A cobertura televisiva da pandemia de Covid-19 em Portugal: um estudo exploratório. Media \& Jornalismo, 20(37), Artigo 185204. https://doi.org/g7cs

Cinelli, M., Quattrociocchi, W., Galeazzi, A., Valensise, C. M., Brugnoli, E., Schmidt, A. L., \& Scala, A. (2020). The COVID-19 social media infodemic. Scientific Reports 10, Artigo 16598. https://doi.org/ghhx8d

Cohen, J. (1988). Statistical power analysis for the behavioral sciences (2. ${ }^{a}$ ed.). Lawrence Erlbaum Associates.

Cohen, J., Cohen, P., West, S., \& Aiken, L. (2003). Applied multiple regression/correlation analysis for the behavioral sciences ( $3^{\mathrm{a}}$ ed.). Lawrence Erlbaum Associates.

Dey, B. L., Al-Karaghouli, W., \& Muhammad, S. S. (2020). Adoption, adaptation, use and impact of information systems during pandemic time and beyond: Research and managerial implications. Information Systems Management, 37(4), 298-302. https://doi.org/g7cq

Dhir, A., Yossatorn, Y., Kaur, P., \& Chen, S. (2018). Online social media fatigue and psychological wellbeing-A study of compulsive use, fear of missing out, fatigue, anxiety and depression. International Journal of Information Management, 40, 141-152. https://doi.org/gd4rdb

Edelman. (2020). Edelman trust barometer 2020. Edelman Trust Barometer - Annual Global Study. Edelman.com. 
Elvestad, E., Phillips, A., \& Feuerstein, M. (2018). Can trust in traditional news media explain Cross-National differences in news exposure of young people online? Digital Journalism, 6(2), $216-235$. https://doi.org/gmsbft

Freundt, T. (2009). El manejo de la confianza y la credibilidade periodisticas como activos intangibles en las empresas de communication. Folios, 18(20), 83-93.

Ghebreyesus, T. (2020, February 15). Detail. World Health Organization. https://bit.ly/3qV4rAs

Gradim, A. (2015). A televisão no seu labirinto. Em P. Serra, S. Sá, \& W. Sousa Filho (Ed.), A televisão Ubíqua (pp. 69-82). LivrosLabcom. https://bit.ly/3qUUEKn

Hays, W. L. (1963). Statistics for psychologists. Holt, Rinehart and Winston.

Hossain, M. M., Sultana, A., \& Purohit, N. (2020). Mental health outcomes of quarantine and isolation for infection prevention: A systematic umbrella review of the global evidence. Epidemiology and Health, PsyArXiv Preprints, 42, Artigo e2020038. https://doi.org/g6j4

Hua, J., \& Shaw, R. (2020). Corona virus (COVID-19) "Infodemic" and emerging issues through a data lens: The case of China. International Journal of Environmental Research and Public Health, 17(7), Artigo 2309. https://doi.org/ggq724

Hung, S. C., Yang, S. C., \& Luo, Y. F. (2021). New media literacy, health status, anxiety, and preventative behaviors related to COVID-19: A cross-sectional study in Taiwan. International Journal of Environmental Research and Public Health, 18(21), Artigo 11247. https://doi.org/g7cv

Hutcheson, G. D., \& Sofroniou, N. (1999). The multivariate social scientist: Introductory statistics using generalized linear models. SAGE Publications Ltd.

Kalogeropoulo, A., Suiter, J., Linards, U., \& Eisenegger, M. (2019). News media trust and news consumption: Factors related to trust in news in 35 countries. International Journal of Communication, 13(22), 3672-3693. https://bit.ly/30JFnkw

Kovach, B., \& Rosenstiel, T. (2005). Os elementos do jornalismo: O que os profissionais do jornalismo devem saber e o público deve exigir. Porto Editora.

Lewis, S. C. (2020). Lack of trust in the news media, institutional weakness, and relational journalism as a potential way forward. Journalism, 21(3), 345-348. https://doi.org/gjmjzk

Luhmann, N. (2021). Realidade dos meios de comunicação. Paulus.

Marktest - Estudos de Mercado, Audiências, Marketing Research, Media. (2020, 24 março). Quarentena impulsiona sites nacionais. (2020, March 24). https://bit.ly/3x2DZpi

Newman, N., Fletcher, R., Kalogeropoulos, A. \& Nielsen, R. (2019). Reuters institute digital news. Digital news report 2019. University of Oxford. https://bit.ly/3HGVX5C

Newman, N., Fletcher, R., Schulz, A., Andı, S. \& Nielsen, R. (2020). Reuters institute digital news. Report 2020. Reuters Institute. University of Oxford. https://bit.ly/3kVEz3k

Park, S., Fisher, C., Flew, T., \& Dulleck, U. (2020). Global mistrust in news: The impact of social media on trust. International Journal on Media Management, 22(2), 83-96. https://doi.org/gh4g2d

Potter, J. W. (2012). Media effects. SAGE Publications, Inc.

Quintanilha L., T., Silva T., M., \& Lapa, T. (2019). Fake news and its impact on trust in the news. Using the Portuguese case to establish lines of differentiation. Communication \& Society, 32(3), 17-33. https://doi.org/gh4fbv

Reardon, S. (2015). Ebola's mental-health wounds linger in Africa. Nature, 519(7541), $13-14$. https://doi.org/gk5b74

Rosen, J. B., \& Schulkin, J. (1998). From normal fear to pathological anxiety. Psychological Review, 105(2), 325350. https://doi.org/czp7sz

Rubin, G. J., \& Wessely, S. (2020). The psychological effects of quarantining a city. BMJ, $363, \mathrm{~m} 313$. https://doi.org/ggpxb4

Salvaterra, F., \& Chora, M. (2021). Relatório de investigação. https://bit.ly/3nB5vGo

Saud, M., Mashud, M., \& Ida, R. (2020). Usage of social media during the pandemic: Seeking support and awareness about COVID-19 through social media platforms. Journal of Public Affairs, 20(4), Artigo e02417. https://doi.org/gktfwx 
Schmitt, J. B., Debbelt, C. A., \& Schneider, F. M. (2017). Too much information? Predictors of information overload in the context of online news exposure. Information, Communication \& Society, 21(8), 1151-1167. https://doi.org/gmcfdk

Shu, K., Sliva, A., Wang, S., Tang, J., \& Liu, H. (2017). Fake news detection on social media. ACM SIGKDD Explorations Newsletter, 19(1), 22-36. https://doi.org/gf6d5n

Stamm, K., Johnson, M., \& Martin, B. (1997). Differences among newspapers, television, and radio in their contribution to knowledge of the contract with america. Journalism \& Mass Communication Quarterly, 74(4), 687-702. https://doi.org/dgs43r

Stratton, J. (2018). Death and the spectacle in television and social media. Television \& New Media, 21(1), 3-24. https://doi.org/gh8gjb

Tabachnick, B., \& Fidell, L. (2007). Using multivariate statistics. Pearson Education, Inc.

Taylor, S. (2020). The psychology of pandemics: Preparing for the next global outbreak of infectious disease. Cambridge Scholars Publishing.

Trindade, I. A., \& Ferreira, N. B. (2020). COVID-19 pandemic's effects on disease and psychological outcomes of people with inflammatory bowel disease in Portugal: A preliminary research. Inflammatory Bowel Diseases, 27(8), 1224-1229. https://doi.org/g6j5

Wang, Y., McKee, M., Torbica, A., \& Stuckler., D. (2019). Systematic literature review on the spread of healthrelated misinformation on social media. Social Science and Medicine, 240, Artigo 112552. https://doi.org/gf8kht

Wang, Y., Zhao, X., Feng, Q., Liu, L., Yao, Y., \& Shi, J. (2020). Psychological assistance during the coronavirus disease 2019 outbreak in china. Journal of Health Psychology, 25(6), 733-737. https://doi.org/ggv479

Wilder-Smith, A., \& Freedman, D. O. (2020). Isolation, quarantine, social distancing and community containment: Pivotal role for old-style public health measures in the novel coronavirus (2019-nCoV) outbreak. Journal of Travel Medicine, 27(2), 27. https://doi.org/ggpw9w

Wolf, M. (2006). Teorias da comunicação. Editorial Presença.

Ytre-Arne, B., \& Moe, H. (2021). Doomscrolling, monitoring and avoiding: news use in COVID-19 pandemic lockdown. Journalism Studies, 1-17. https://doi.org/g7cr 